\title{
Low Metabolite Cost and Air-Tight Leaf Structure Regulated by Multiple Loci Enhance Rice Biomass
}

Ichiro Kasajima

Laboratory of Plant Pathology, Department of Agriculture, Iwate University, Ueda 3-18-8, Morioka, Iwate 0208550, Japan

kasajima2008@live.jp

Abstract:Improvement of rice growth is directly connected to solution of food shortage. In spite of enormous research efforts, specific physiological mechanisms and genes that increase rice growth is not completely clear. In this study, clear difference in growth rates of rice cultivars was identified. Part of Indica cultivars (Kasalath and Nona Bokra) grew bigger, compared with the other cultivars such as Koshihikari. Measurement of growth of CSSL lines between Koshihikari and Nona Bokra, or BIL lines between Koshihikari and Kasalath indicated that these vigorous growth phenotypes are caused by combination of multiple loci. Transpiration rate of Nona Bokra was much lower than Koshihikari. Metabolite contents, such as histidine, valine, glutathione and phosphoenol pyruvate were significantly lower in Nona Bokra. These observations suggested involvement of air tightness and low metabolite content in rapid growth and greater biomass of these Indica cultivars.

Keywords: Amino Acid, Biomass, Metabolite, Rice, Transpiration INTRODUCTION

In spite of ever growing population in the world, production of rice, one of the most important staple crops, suffer from limitations in enhancement of production (Nguyen 2002). Balance between carbohydrate 'source' and 'sink' have been proposed to be one of the keys to enhance crop production. Through increase in number and size of rice grains, sink size is successfully improved and rice production is enhanced, by introduction of specific genes (Ishimaru et al. 2013; Fujita et al. 2013). On the contrary, improvement of source capacity, such as photosynthetic rate, seems to be more difficult. There would be no successful example of source improvement in rice yet.

Part of the difficulty in analysis of photosynthesis seems to lie in quite variable rates of photosynthesis, measured by gas-exchange analyzer. Photosynthesis rate is variable even in the same leaf, at different positions, under different conditions such as light intensity, humidity, and temperature, and at different time of a day. In comparative analysis between cultivars, slight difference in photosynthesis rate results in large different in biomass. Mathematically, biomass is the integral of photosynthesis rate. Loss of carbohydrate by respiration and root exudations also have to be taken into account. Then, it may be impossible to totally analyze and compare slight difference in these factors affecting net photosynthesis rate.

Result of integrations of all these cellular processes are measurable with the simplest method: biomass, or dry weight. In this study, biomass of rice genetic resources was measured to obtain overview of genetic mechanisms regulating rice biomass in different cultivars. Together with morphological and physiological analyses, this study provides novel insight into the mechanism of photosynthetic source enhancement in rice cultivars.

\section{MATERIALS AND METHODS}

\section{Plant Materials and Growth Conditions}

Rice cultivars, CSSLs and BILs were obtained from NIAS (National Institute of Agrobiological Sciences, Japan). Rice seeds were germinated in water at $25^{\circ} \mathrm{C}$ for five days. Seedlings were transferred to plastic pots, which were filled with bon-sol \#1 (Sumitomo Chemical, Tokyo, Japan). Plants were grown in sun-lit greenhouse at 
$60 \%$ relative humidity, with $28^{\circ} \mathrm{C}$ (day) $/ 24^{\circ} \mathrm{C}$ (night) temperature controls. In the analysis of BIL lines, 44 representative lines harboring relatively small ratio of genome substitution were cultivated. Only 26 of these lines grew healthy in greenhouse.

\section{Measurement of Transpiration Rate}

Leaf blades were excised from rice plants. Weights of leaf blades were measured with electric scale at indicated time points.

\section{Preparation of Leaf Replica}

Chemical bond (cemedine: Cemedine, Tokyo, Japan) was pasted on leaves. After bond is solidified, it was removed from leaves and observed with light microscope.

\section{Measurement of Metabolite Contents}

Metabolites were measured with CE/MS system (Agilent Technologies, Santa Clara, USA) as reported previously (Takahara et al. 2010).

\section{RESULTS AND Discussion}

\section{Identification of Vigorously Growing Rice Cultivars}

During studies on rice cultivars, I noticed clear difference in growth rate between rice cultivars. For example, Indica cultivar Nona Bokra grows much bigger than Japonica cultivar Koshihikari (Figure 1A, 1B). Total fresh weights of five Japonica cultivars (Nipponbare, Koshihikari, Akihikari, Sasanishiki and Asominori) and four Indica cultivars (Kasalath, Habataki, Nona Bokra and IR24) are shown in Figure 1C. Among these cultivars, fresh weights of Kasalath and Nona Bokra were much greater than the other cultivars.

In order to characterize genetic loci that regulate higher biomass in Nana Bokra and Kasalath, biomass of CSSLs (chromosome segment substitution lines) between Koshihikari and Nona Bokra (Figure 1D), or BILs (backcross inbred lines) between Koshihikari and Kasalath (Figure 1E) were measured. As a result of repetitive crosses and genetic selection, only small portion of whole Koshihikari genome is substituted by genomes of Nona Bokra or Kasalath in CSSL and BIL lines (Takai et al. 2007; Ma et al. 2002). Against expectation, plant biomass of all CSSL lines was similar to Koshihikari, and no CSSL line had large biomass like Nona Bokra. In contrast, three BIL lines between Koshihikari and Kasalath showed much higher biomass than Koshihikari. Line numbers of these lines are KK-BIL-072, 102 and 131. Genome structures of these three BIL lines are illustrated in Figure 1F.

\section{Lower Transpiration Rate in Nona Bokra}

Rate of photosynthesis is regulated by multiple physiological factors, such as rate of gas exchange between inside and outside of leaves, activity of RubisCO enzyme, and concentration of RubisCO substrate. In this study, I focused on rate of gas exchange. Rate of gas exchange was measured by transpiration rate, which is estimated by weight loss of excised leaf blades (Figure 2A). In both Koshihikari and Nona Bokra, transpiration was slower in fourth leaves than in third leaves. In addition, transpiration was clearly slower in Nona Bokra than in Koshihikari.

Difference in transpiration rate may be caused by difference in stomatal density, stomatal size, or stomatal aperture. Stomatal aperture of rice leaves is difficult to compare, but there wasn't discernible difference in stomatal density in adaxial or abaxial side of Koshihikari and Nona Bokra leaves (Figure 2B through 2E). On the other hand, width of stomata was narrower in Nona Bokra leaves than in Koshihikari leaves (Figure 2F, 2G). Difference in transpiration rate between third and fourth leaves (Figure 2A) associated negative correlation between leaf size and transpiration rate, i.e. bigger leaves may have higher transpiration rate per leaf weight. Figure $2 \mathrm{H}$ is the plot of transpiration rate against leaf fresh weight. As expected, there was negative correlation between transpiration rate and leaf weight. Yet, this does not mean that bigger leaves have lower surface area 
per weight: different leaf positions of both Koshihikari and Nona Bokra had similar values of specific leaf area (Figure 2I). Consistent with lower transpiration rate, water content was higher in Nona Bokra than in Koshihikari, and higher in fourth leaves than in third leaves (Figure 2J).

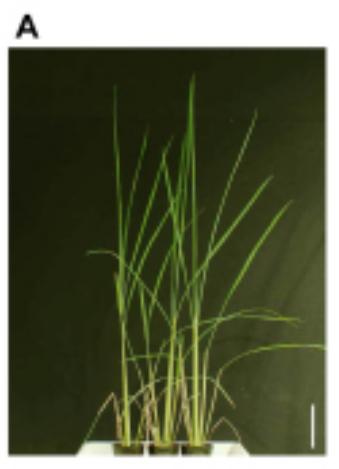

B

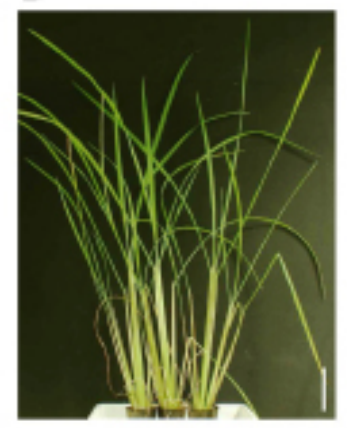

C

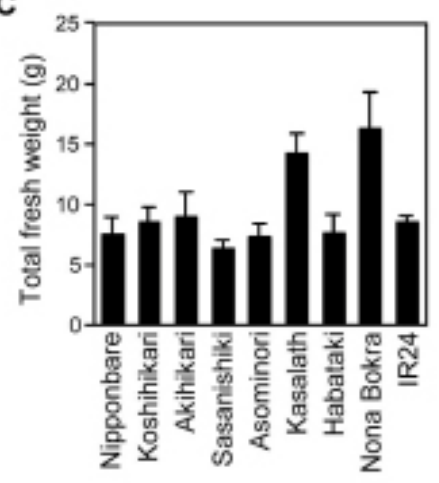

D

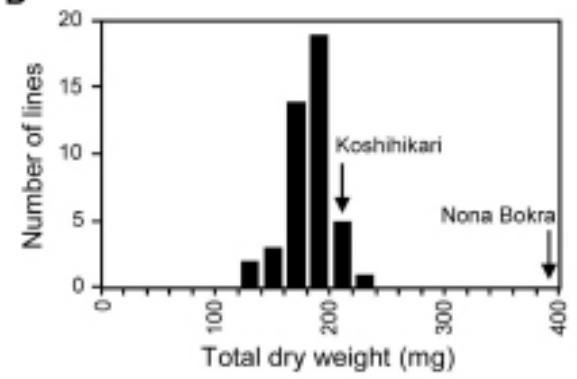

E

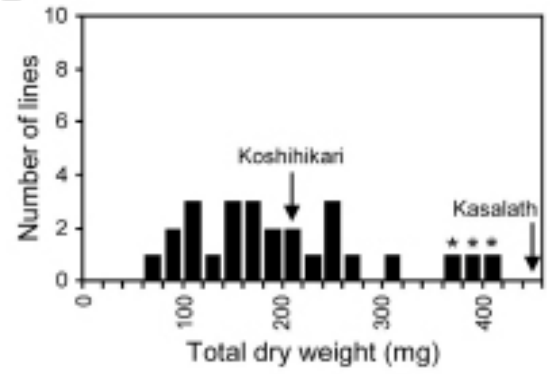

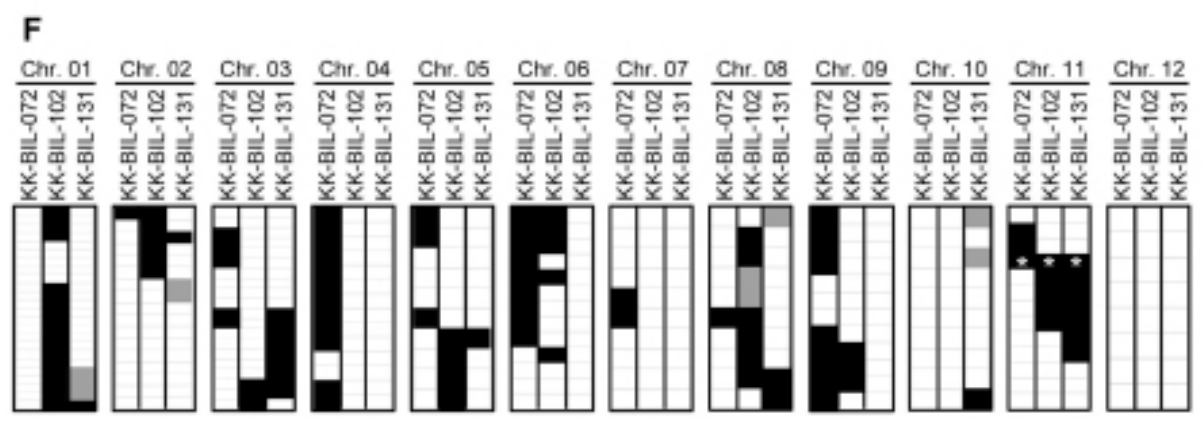

Fig1. Measurement of rice biomass.

(A) Koshihikari plants grown for 60 days in plastic pots and (B) Nona Bokra plants grown for the same period. Bars $=10 \mathrm{~cm}$. (C) Total fresh weight (including root) of nine cultivars, after growth in plastic pot for 61 days. $\mathrm{n}=$ 4. Data represent means and standard deviations. (D) Biomass of 44 CSSL lines between Koshihikari and Nona Bokra, after growth for 30 days. (E) Biomass of 26 BIL lines between Koshihikari and Kasalath, after growth for 35 days. Three high-biomass BILs are indicated with asterisks. (F) Schematic representation of genome structure in three high-biomass BIL lines. White represents Koshihikari genotype, and black represents Kasalath genotype. Gray parts are heterozygous. White asterisks represent the common substituted locus (genetic marker G320), among three BILs.

\section{Lower Metabolite Contents in Nona Bokra}

To identify possible difference in metabolism, amino acid and organic acid contents in leaves were compared between Koshihikari and Nona Bokra (Figure 3A, 3B). Metabolite contents tended to be lower in Nona Bokra 
leaves. Contents of seven amino acids (histidine, citrulline, isoleucine, leucine, valine, phenylalanine and homoserine), both reduced and oxidized forms of glutathione, phosphoenolpyruvate and 3-phosphoglycerate were significantly lower in Nona Bokra, and no metabolite content was significantly higher in Nona Bokra.

Genetic Regulation of Biomass in Rice

A

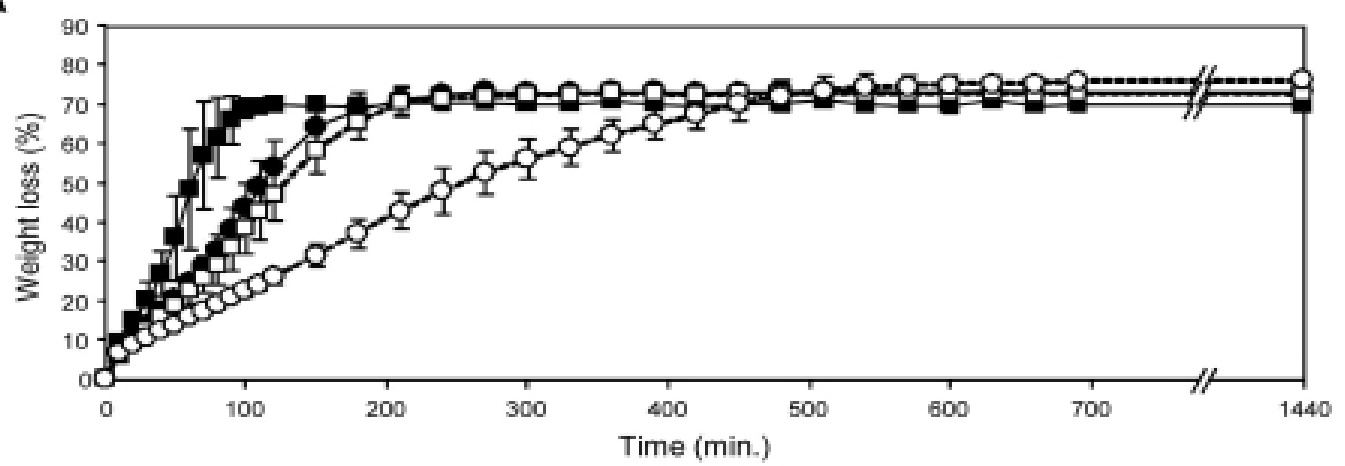

B

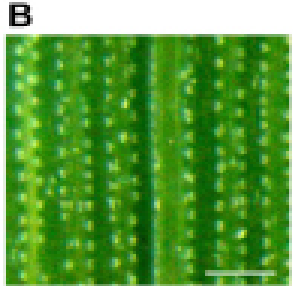

$\mathbf{F}$

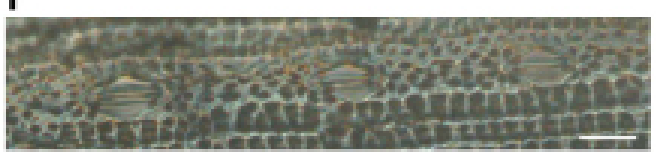

H

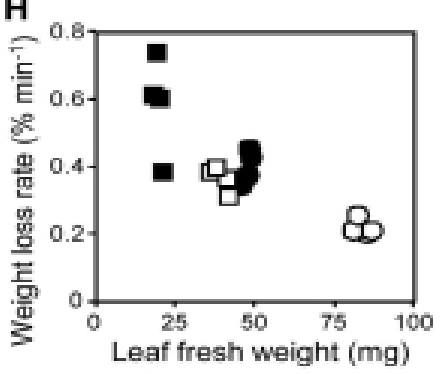

C

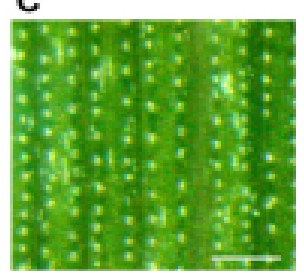

I
D

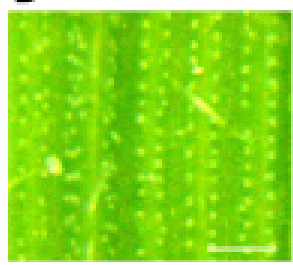

G
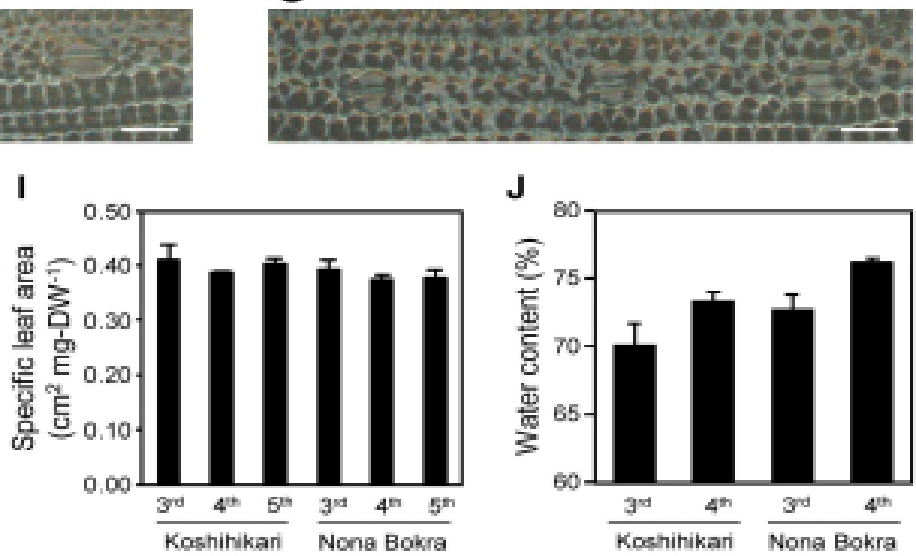

E

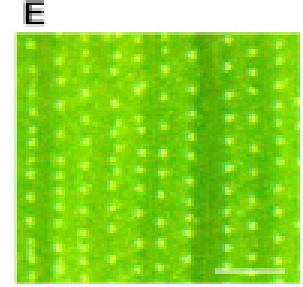

$\mathrm{J}$

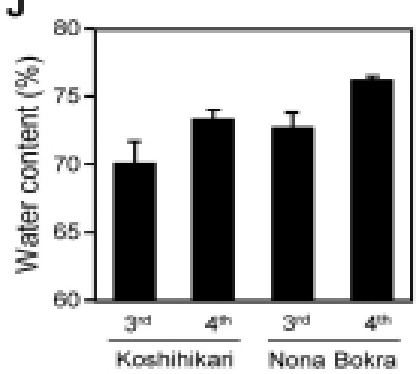

Fig2. Measurement of transpiration rate.

(A) Transpiration rate of rice leaves, measured by 'percent weight loss'. Data represent Koshihikari third leaf (filled square), Koshihikari fourth leaf (filled circle), Nona Bokra third leaf (open square), and Nona Bokra fourth leaf (open circle). $\mathrm{n}=4$. Data represent means and standard deviations. (B to E) Stereomicroscope images of rice leaves (three weeks grown in pot): adaxial side of Koshihikari leaf blade (B), abaxial side of Koshihikari (C), adaxial side of Nona Bokra (D), and abaxial side of Nona Bokra (E). Bars $=200 \mu \mathrm{m}$. Stomata are visible as white spots. (F and G) Microscopic image of leaf replica: adaxial side of Koshihikari (F) and Nona Bokra (G). Bars = 20 $\mu \mathrm{m}$. (H) Relationship between leaf size and transpiration rate (during 10-20 minutes after excision). Squares and circles represent same leaves as (A). (I) Specific leaf area. $n=4$. (J) Water content of leaves. $n=4$. Plants were grown for four weeks in (A), (H), (I) and (J). 
A gene called OsEBS derived from wild rice is reported to increase biomass of cultivated rice (Dong et al. 2013). Unlike wild rice, genetic analysis of biomass regulation in cultivated rice in this study did not identify a single major locus (Figure 1). Absence of CSSL line with higher biomass than Koshihikari suggests that multiple loci cooperatively function and increase rice biomass. In contrast to CSSL, in which only a part of one chromosome is substituted by genome of other cultivar, multiple parts of genome are substituted by donor cultivar in BIL. Analysis of BIL lines identified three high-biomass lines (Figure 1E). Substituted parts of these three BILs are different from each other, except for a locus in chromosome 11. Nevertheless, BILs that have substitutions in this locus are not necessarily bigger than Koshihikari. The three high-biomass BILs will have enhanced biomass by combination of independent multiple genetic loci, derived from Kasalath.

\section{Physiological Characteristics of High-Biomass Rice}

High-biomass cultivar Nona Bokra had much lower transpiration rate than Koshihikari (Figure 2A). This result was never expected, under the hypothesis that high-biomass rice should have higher photosynthesis rate.
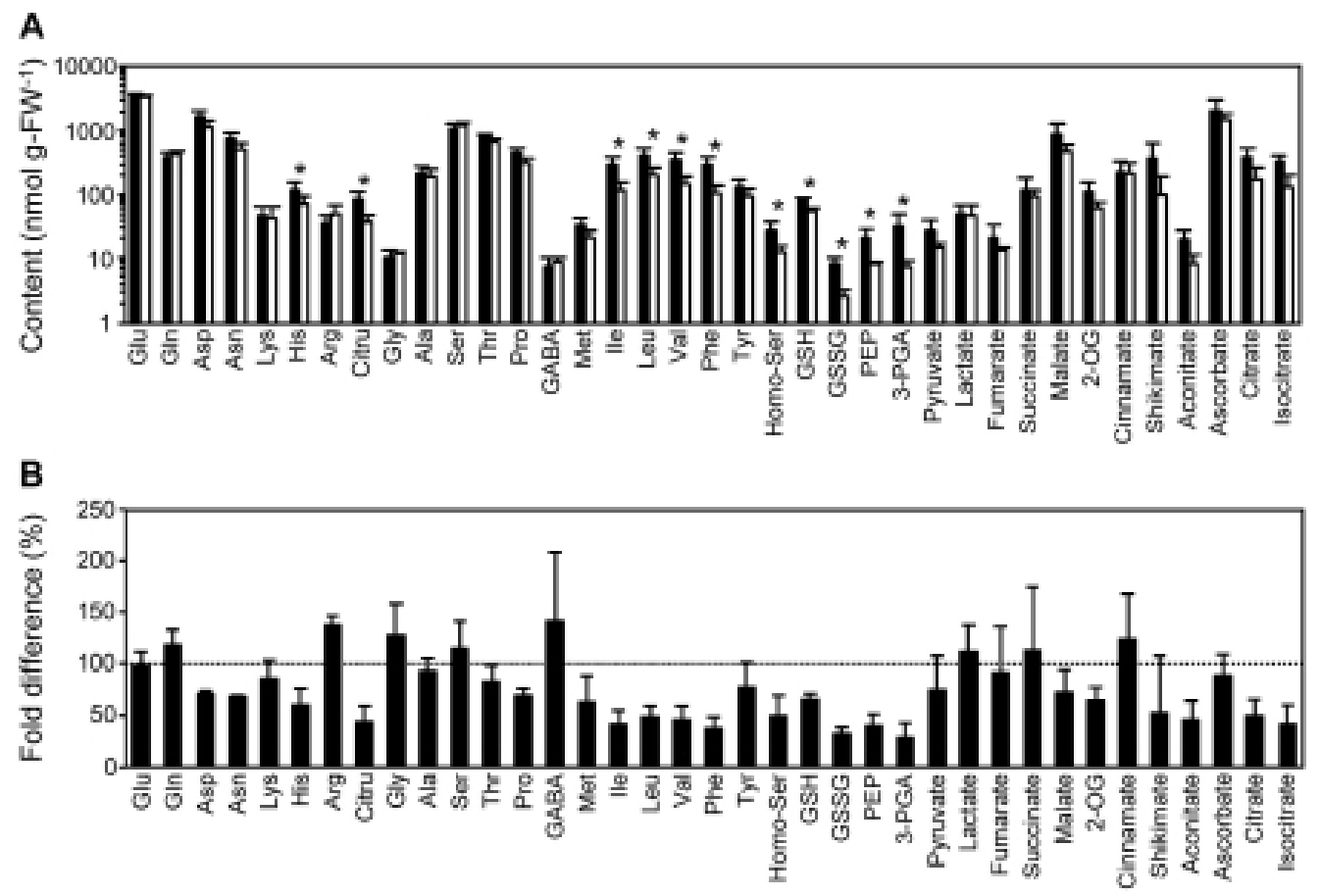

Fig3. Measurement of metabolite contents

(A) Metabolite contents in Koshihikari (black bar) and Nona Bokra (white bar) fifth leaves, excised from plants after grown for four weeks. $n=4$. Data represent means and standard deviations. Asterisks indicate statistical difference by Student's t-test $(\mathrm{P}<0.05)$. (B) Fold difference (Nona Bokra / Koshihikari) in metabolite contents shown in $(\mathrm{A}) . \mathrm{n}=4$. Data represent means and standard deviations.

On the other hand, this observation is quite consistent with a previous report about rice biomass (Jahn et al. 2011). In this report rice cultivars with different biomass was compared, like the study here, and they observed negative correlation between rice biomass and photosynthesis rate. Based on the observation, it would be suggested that low photosynthesis rate in high-biomass rice cultivar is caused by low gas-exchange rate. This low gas-exchange rate seems to be caused by narrower stomata, but the rate of cuticle transpiration also has to be measured in the future. Lower transpiration rate results in higher water content in rice leaves, then possible 
healthier conditions of mesophyll cells and efficient (low-cost) metabolism.

Efficient metabolism in high-biomass rice will be supported by the analysis of metabolite contents (Figure 3). Lower metabolite contents in Nona Bokra suggests that Nona Bokra cell can manage to grow and perform photosynthesis with lower concentrations of metabolic resources in the cell. Most of the seven amino acids whose concentrations were lower in Nona Bokra are synthesized in different metabolic pathways, thus Nona Bokra is predicted to lower amino acid contents through simultaneously downregulating different enzymes. Concentration of glutathione, one of the key anti-oxidants in plant cell, was lower in Nona Bokra. Highly efficient metabolism which can lead to high biomass of Nona Bokra, may cause lower stress tolerance at the same time.

\section{CONCLUSION}

In conclusion, it is deduced that high-biomass rice cultivars develop air-tight leaf structure to restrict transpiration rate and protect mesophyll cells from dryness, then enhances effective metabolism. Genetic mechanism of this regulation is complicated, and requires further experiments.

\section{REFERENCES}

1. Dong X, Wang X, Zhang L, Yang Z, Xin X, Wu S, Sun C, Liu J, Yang J, Luo X. Identification and characterization of OsEBS, a gene involved in enhanced plant biomass and spikelet number in rice. Plant Biotechnol J. 2013; 11:1044-57.

2. Fujita D et al. NAL1 allele from a rice landrace greatly increases yield in modern indica cultivars. Proc Natl Acad Sci USA. 2013; 110:20431-6.

3. Ishimaru K, Hirotsu N, Madoka Y, Murakami N, Hara N, Onodera H, Kashiwagi T, Ujiie K, Shimizu B, Onishi A, Miyagawa H, Katoh E. Loss of function of the IAA-glucose hydrolase gene TGW6 enhances rice grain weight and increases yield. Nat Genet. 2013; 45:707-11.

4. Jahn CE, Mckay JK, Mauleon R, Stephens J, McNally KL, Bush DR, Leung H, Leach JE. Genetic variation in biomass traits among 20 diverse rice varieties. Plant Physiol. 2011; 155:157-68.

5. Ma JF, Shen RF, Zhao ZQ, Wissuwa M, Takeuchi Y, Ebitani T, Yano M. Response of rice to Al stress and identification of quantitative trait loci for Al tolerance. Plant Cell Pysiol. 2002; 43:652-9.

6. Nguyen VN. Rice production, consumption and nutrition. In FAO Rice Information. 3rd volume: Food and Agriculture Organization of the United Nations; 2002.

7. Takahara K, Kasajima I, Takahashi H, Hashida S, Itami T, Onodera H, Toki S, Yanagisawa S, Kawai-Yamada M, Uchimiya H. Metabolome and photochemical analysis of rice plants overexpressing Arabidopsis NAD kinase gene. Plant Physiol. 2010; 152:1863-73.

8. Takai T, Nonoue Y, Yamamoto S, Yamanouchi U, Matsubara K, Liang ZW, Lin H, Ono N, Uga Y, Yano M. Development of chromosome segment substitution lines derived from backcross between indica donor rice cultivar 'Nana Bokra' and japonica recipient cultivar 'Koshihikari'. Breed Sci. 2007; 57:257-61.

Citation: Ichiro Kasajima Laboratory of Plant Pathology, Department of Agriculture, Iwate University, Ueda 3-18-8, Morioka, Iwate 020-8550, Japan. Low Metabolite Cost and Air-Tight Leaf Structure Regulated by Multiple Loci Enhances Rice Biomass American Research Journal of Biosciences Volume 2016; pp:1-6

Copyright (C) 2016 Ichiro Kasajima This is an open access article distributed under the Creative Commons Attribution License, which permits unrestricted use, distribution, and reproduction in any medium, provided the original work is properly cited. 